\title{
Research Article \\ Optimal Selling of an Asset under Incomplete Information
}

\author{
Erik Ekström and Bing Lu \\ Department of Mathematics, Uppsala University, P.O. Box 480, 75106 Uppsala, Sweden \\ Correspondence should be addressed to Erik Ekström, ekstrom@math.uu.se
}

Received 5 August 2011; Accepted 20 October 2011

Academic Editor: Tusheng Zhang

Copyright (C 2011 E. Ekström and B. Lu. This is an open access article distributed under the Creative Commons Attribution License, which permits unrestricted use, distribution, and reproduction in any medium, provided the original work is properly cited.

We consider an agent who wants to liquidate an asset with unknown drift. The agent believes that the drift takes one of two given values and has initially an estimate for the probability of either of them. As time goes by, the agent observes the asset price and can therefore update his beliefs about the probabilities for the drift distribution. We formulate an optimal stopping problem that describes the liquidation problem, and we demonstrate that the optimal strategy is to liquidate the first time the asset price falls below a certain time-dependent boundary. Moreover, this boundary is shown to be monotonically increasing, continuous and to satisfy a nonlinear integral equation.

\section{Introduction}

This paper treats the problem of optimal timing for an irreversible sale of an indivisible asset under incomplete information about its drift. The asset price is assumed to follow a geometric Brownian motion $X$ with unknown drift, and an agent who decides to sell at time $t$ receives at this time the amount $X_{t}$. The objective of the agent is to choose a liquidation time for which the expected value of the (discounted) asset price is maximised. Such problems are important for all types of investors with insufficient knowledge of the future trend of an asset.

In the case with complete information about the model parameters of $X$, the corresponding optimal liquidation problem is trivial. Indeed, if the drift is larger than the interest rate, then on average the asset price grows faster than money in a risk-free bank account, and the agent should keep the asset as long as possible. Similarly, a drift smaller than the interest rate implies that the agent should liquidate the asset immediately, and instead deposit the money in the bank. However, we remark that the assumption of complete information about the parameters of $X$ is quite strong. While the volatility of an asset, at least in principle, can be estimated instantaneously by observing the price fluctuations over an arbitrarily short time period, the drift is notoriously difficult to estimate from historical data. In fact, to achieve 
a decent accuracy in the estimate for the drift, one typically needs observations of the process from hundreds of years.

Instead, we allow for incomplete information by modelling the drift as a random variable which is not directly observable for the agent. Initially, the agent's beliefs about the drift are summarised by a probability distribution. As time goes by, however, he observes the asset price process, and based on these observations his beliefs may change. Naturally, if the asset price rises quickly, then the agent will consider it more likely that the drift takes the larger of the two values. Consequently, he would in this case postpone the liquidation. Similarly, if the asset falls drastically, then it is likely that the true drift is small, and the agent would be more inclined to liquidate his position early. We show below that this intuition is true; that is, there exists a boundary between the continuation region and the stopping region such that the optimal liquidation time coincides with the first time the asset price falls below this boundary. We also derive monotonicity and continuity properties of the boundary, and we show that it satisfies a nonlinear integral equation similar to the one which characterises the optimal stopping boundary for the American put option.

Related problems of liquidating an indivisible asset have been studied in $[1,2]$. These papers study a risk-averse agent who wants to sell an indivisible asset with the possibility of hedging some of the risk by investing in a correlated stock market. The paper [3], see also [4], studies a problem of optimal selling of an asset, where optimality is measured by closeness between the current asset price and its ultimate maximum over the whole time period. In all the papers referred to above, the agent is assumed to have complete information about the underlying price processes. The methods we use to treat the incomplete information in our setting are standard and based on filtering theory see, for example, [5]. An early application of these techniques is the sequential testing of two alternative hypotheses about the drift of a Brownian motion; for further details and related references, see Chapter VI.21 in [6]. Similar techniques to tackle investment problems in markets with incomplete information are also applied in $[7,8]$, where the problem of maximising expected utility of terminal wealth by trading in different assets is studied. The papers $[9,10]$ study the optimal timing for an investment under incomplete information. Mathematically, the investment problem in [9] is equivalent to the pricing of an American call option written on an asset with unknown drift. Using filtering techniques, the problem is reduced to an optimal stopping problem with complete information, but with two underlying spatial dimensions. A clever observation in [10] reduces the two-dimensional problem into a one-dimensional optimal stopping problem, but in general for a time-dependent payoff function (for one specific choice of parameters, however, the time dependence disappears and the optimal stopping problem can be solved explicitly). In the present paper, the optimal liquidation problem has a linear payoff, which implies that the problem can be reduced to a one-dimensional optimal stopping problem for a time homogeneous diffusion with an affine payoff regardless of what parameters are chosen. Consequently, this reduced problem is straightforward to analyse using standard methods from optimal stopping theory.

The present paper is organised as follows. In Section 2 we formulate the liquidation problem with incomplete information, and we apply filtering techniques to write it as a twodimensional problem with complete information. Moreover, we apply a Girsanov transformation that reduces the problem to a one-dimensional optimal stopping problem for a time homogeneous diffusion with an affine payoff function. We also provide the solution of the optimal liquidation problem (in terms of the boundary of the auxiliary optimal stopping problem); see Theorem 2.5. The auxiliary optimal stopping problem is treated in Section 3, where we demonstrate the existence of a monotonically increasing and continuous optimal 
stopping boundary. We also show that the boundary together with the value function solves a parabolic free boundary problem. In Section 4 we derive an integral equation for the optimal stopping boundary. Finally, in Section 5 we study a related situation in which the agent seeks an optimal time to close a short position in the asset.

\section{The Optimal Liquidation Problem and Its Solution}

To model the situation with incomplete information, we assume that the asset price process $X$ follows a geometric Brownian motion with unknown drift $\mu$ and constant volatility $\sigma>0$. More precisely,

$$
d X_{t}=\mu X_{t} d t+\sigma X_{t} d \widetilde{W}_{t}, \quad t \geq 0
$$

where $\widetilde{W}$ is a standard Brownian motion independent of $\mu$ on a probability space $(\Omega, \mathcal{F}, P)$. Here, for simplicity, we assume that the drift $\mu$ can only take two values $\mu_{h}$ and $\mu_{l}$ satisfying $\mu_{l}<r<\mu_{h}$, where the interest rate $r \geq 0$ is a constant, and the initial asset price $X_{0}$ is a positive constant. We consider an agent who owns the asset and wants to liquidate his position before a given future fixed time $T>0$. At the initial time 0 , the true value of the drift $\mu$ is not known, but we assume that the agent has an initial guess for the probabilities of the events $\left\{\mu=\mu_{l}\right\}$ and $\left\{\mu=\mu_{h}\right\}$. More explicitly, we assume that the agent's initial estimate of the probability of the event $\left\{\mu=\mu_{h}\right\}$ is a constant $\Pi_{0} \in(0,1)$. Accordingly, the estimate of the probability of $\left\{\mu=\mu_{l}\right\}$ is $1-\Pi_{0}$. Furthermore, we assume that the agent can observe the value process $X$, but neither the drift $\mu$ nor the Brownian motion $\widetilde{W}$. This is a natural assumption since, in a real world situation, no underlying Brownian motion can be observed, and to estimate the drift with a high precision is infeasible.

Example 2.1. Consider a Brownian motion $Z_{t}=a t+b B_{t}$ with drift $a$ and volatility $b$ (here $B$ is a standard Brownian motion). An estimate for the drift $a$ based on observations over the time period $[0, t]$ would be $\widehat{a}=Z_{t} / t$, and a $95 \%$ confidence interval is then given by $(\widehat{a}-1.96 b / \sqrt{t}, \hat{a}+1.96 b / \sqrt{t})$. Even if the volatility is small, say $b=0.1$, in order for the confidence interval to be reasonably tight, say $(\widehat{a}-0.02, \widehat{a}+0.02)$, one needs approximately 100 years of observations! Moreover, the observation time that is needed grows (inverse) quadratically in the length of the confidence interval.

The objective of this paper is to determine when to sell the stock in order to maximise the expected wealth. More precisely, let $\left\{\mathcal{F}_{t}^{X}\right\}_{t \in[0, T]}$ be the completion of the filtration generated by the process $X$. The agent then seeks an $\mp^{X}$-stopping time $\tau$ with $0 \leq \tau \leq T$ for which the supremum

$$
V=\sup _{0 \leq \tau \leq T} E\left[e^{-r \tau} X_{\tau}\right]
$$

is attained, where the supremum is taken over $\mp^{X}$-stopping times $\tau$.

Remark 2.2. Note that in the omitted cases $\Pi_{0}=0$ and $\Pi_{0}=1$, the problem is simply a problem with complete information, and the solution is trivial. Indeed, if $\Pi_{0}=1$, then $\mu=\mu_{h}$ and $e^{-r t} X_{t}$ is a submartingale, so optional sampling yields that $V=X_{0} e^{\left(\mu_{h}-r\right) T}$. Similarly, if $\Pi_{0}=0$, then $e^{-r t} X_{t}$ is a supermartingale and $V=X_{0}$. Also note that it is necessary to have $T<\infty$ in order 
to avoid a degenerate problem when $\Pi_{0}>0$. In fact, plugging in the stopping time $\tau=n$ and letting $n$ tend to infinity shows that in the perpetual case we would have an infinite value $V$.

Remark 2.3. Inserting $\tau=0$ into (2.2) yields a lower bound $V \geq X_{0}$. Another lower bound can be found by comparing with the corresponding "European value" $X_{0}\left(\Pi_{0} e^{\left(\mu_{h}-r\right) T}+(1-\right.$ $\left.\Pi_{0}\right) e^{\left(\mu_{l}-r\right) T}$ ) determined by inserting $\tau=T$ in (2.20). Moreover, an upper bound for $V$ can be found by observing that increasing $u_{l}$ to $r$ simply gives a higher payoff. In that case it is clear that $e^{-r t} X_{t}$ is a submartingale, so the optional sampling theorem gives that $V \leq X_{0}\left(\Pi_{0} e^{\left(\mu_{h}-r\right) T}+1-\Pi_{0}\right)$. Consequently,

$$
\max \left\{X_{0}, X_{0}\left(\Pi_{0} e^{\left(\mu_{h}-r\right) T}+\left(1-\Pi_{0}\right) e^{\left(\mu_{l}-r\right) T}\right)\right\} \leq V \leq X_{0}\left(\Pi_{0} e^{\left(\mu_{h}-r\right) T}+1-\Pi_{0}\right) .
$$

Naturally, if $\Pi_{0}$ is small, then the agent is rather confident that the true drift is $u_{l}$, and he would liquidate immediately and rather deposit the money in the bank. On the other hand, if $\Pi_{0}$ is close to one, then he considers it likely that the drift is $u_{h}$, and he would prefer to postpone the selling. By observing the process $X$, however, the agent's estimates for the probabilities of the events $\left\{\mu=\mu_{h}\right\}$ and $\left\{\mu=\mu_{l}\right\}$ may change. For $t \geq 0$, let

$$
\Pi_{t}=P\left[\mu=\mu_{h} \mid \Psi_{t}^{X}\right]
$$

be the probability at time $t$ that $\mu=\mu_{h}$ conditional on the observations of $X$ up to time $t$. From Theorems 7.12 and 9.1 in Liptser and Shiryayev [5], the value process $X$ and the belief process $\Pi$ satisfy

$$
\left(\begin{array}{c}
d X_{t} \\
X_{t} \\
d \Pi_{t}
\end{array}\right)=\left(\begin{array}{c}
\mu_{l}+\Pi_{t}\left(\mu_{h}-\mu_{l}\right) \\
0
\end{array}\right) d t+\left(\begin{array}{c}
\sigma \\
\omega \Pi_{t}\left(1-\Pi_{t}\right)
\end{array}\right) d \bar{W}_{t}
$$

where $\omega=\left(\mu_{h}-\mu_{l}\right) / \sigma$ and $\left(\bar{W}, \mathscr{F}^{X}\right)$ is a $P$-Brownian motion defined by

$$
d \bar{W}_{t}=d \widetilde{W}_{t}+\frac{\mu-\left(1-\Pi_{t}\right) \mu_{l}-\Pi_{t} \mu_{h}}{\sigma} d t
$$

Note that the drift of $X$ depends on $\Pi$, so the optimal stopping problem (2.2) has two underlying spatial dimensions. However, since $X$ and $\Pi$ are both expressed in terms of the same Brownian motion $\bar{W}$, the number of spatial dimensions can be reduced. Indeed, in the following we follow [10] and use a Girsanov transformation to reduce the problem to a onedimensional stopping problem.

To do this, define a new process $W$ by

$$
d W_{t}=\left(\omega \Pi_{t}-\sigma\right) d t+d \bar{W}_{t}
$$


and a new measure $P^{*}$ by its Radon-Nikodym derivative

$$
\begin{aligned}
\frac{d P^{*}}{d P} & =\exp \left\{-\frac{1}{2} \int_{0}^{T}\left(\sigma-\omega \Pi_{t}\right)^{2} d t+\int_{0}^{T}\left(\sigma-\omega \Pi_{t}\right) d \bar{W}_{t}\right\} \\
& =\exp \left\{\frac{1}{2} \int_{0}^{T}\left(\sigma-\omega \Pi_{t}\right)^{2} d t+\int_{0}^{T}\left(\sigma-\omega \Pi_{t}\right) d W_{t}\right\}
\end{aligned}
$$

with respect to $P$. By Girsanov's Theorem, $W$ is a $P^{*}$-Brownian motion. Next, define the likelihood ratio $\Phi$ by $\Phi_{t}=\Pi_{t} /\left(1-\Pi_{t}\right)$. A straightforward application of Ito's formula gives

$$
d \Phi_{t}=\omega^{2} \Pi_{t} \Phi_{t} d t+\omega \Phi_{t} d \bar{W}_{t}
$$

Expressing the dynamics in terms of $W$ gives

$$
\left(\begin{array}{c}
\frac{d X_{t}}{X_{t}} \\
\frac{d \Phi_{t}}{\Phi_{t}}
\end{array}\right)=\left(\begin{array}{c}
\mu_{l}+\sigma^{2} \\
\omega \sigma
\end{array}\right) d t+\left(\begin{array}{c}
\sigma \\
\omega
\end{array}\right) d W_{t}
$$

so both $X$ and $\Phi$ are geometric Brownian motions under $P^{*}$. Moreover, the filtration generated by $W$ coincides with the one generated by $X$.

Define the likelihood process

$$
\eta_{t}=\exp \left\{-\frac{1}{2} \int_{0}^{t}\left(\sigma-\omega \Pi_{s}\right)^{2} d s+\int_{0}^{t}\left(\omega \Pi_{s}-\sigma\right) d W_{s}\right\} .
$$

Since $\left(W, \mathcal{F}^{X}\right)$ is a $P^{*}$-Brownian motion, the process $\eta$ is an $\Psi^{X}$-martingale under $P^{*}$. Let

$$
F_{t}=\frac{1+\Phi_{t}}{1+\Phi_{0}}
$$

Then

$$
\frac{d F_{t}}{F_{t}}=\frac{d \Phi_{t}}{1+\Phi_{t}}=\sigma \omega \Pi_{t} d t+\omega \Pi_{t} d W_{t}
$$

so

$$
F_{t}=\exp \left\{\frac{1}{2} \int_{0}^{t}\left(2 \sigma \omega \Pi_{s}-\omega^{2} \Pi_{s}^{2}\right) d s+\int_{0}^{t} \omega \Pi_{s} d W_{s}\right\}
$$

Consequently,

$$
\eta_{t} X_{t}=e^{\mu_{l} t} F_{t} X_{0}
$$


Denote by $E^{*}$ the expectation operator with respect to the new measure $P^{*}$, and let $\tau \leq T$ be an $\mathcal{F}^{X}$-stopping time. Then

$$
\begin{aligned}
E\left[e^{-r \tau} X_{\tau}\right] & =E^{*}\left[e^{-r \tau} \eta_{T} X_{\tau}\right]=E^{*}\left[e^{-r \tau} \eta_{\tau} X_{\tau}\right] \\
& =X_{0} E^{*}\left[e^{\left(\mu_{l}-r\right) \tau} F_{\tau}\right]=\frac{X_{0}}{1+\Phi_{0}} E^{*}\left[e^{\left(\mu_{l}-r\right) \tau}\left(1+\Phi_{\tau}\right)\right]
\end{aligned}
$$

where the third equality follows by conditioning upon $\Psi_{\tau}^{X}$ together with the martingale property of $\eta$.

Remark 2.4. Note that the measure change defined in (2.8) slightly differs from the one in $[9,10]$, where the new measure instead is defined so that the Radon-Nikodym derivative coincides with

$$
\exp \left\{-\frac{1}{2} \int_{0}^{T} \omega^{2} \Pi_{t}^{2} d t-\int_{0}^{T} \omega \Pi_{t} d \bar{W}_{t}\right\}
$$

on $\boldsymbol{F}_{T}^{X}$. To reduce the number of spatial dimensions, Klein [10] then employs the equality

$$
X_{t}=X_{0} e^{\varepsilon t}\left(\frac{\Phi_{t}}{\Phi_{0}}\right)^{\beta}
$$

where

$$
\beta=\frac{\sigma}{\omega}=\frac{\sigma^{2}}{\mu_{h}-\mu_{l}}, \quad \varepsilon=\frac{\left(\mu_{h}+\mu_{l}-\sigma^{2}\right)}{2} .
$$

If $\varepsilon=0$, then the obtained optimal stopping problem is time homogeneous, and an explicit solution can be found. The measure change in (2.8) is tailormade for the situation of a linear payoff structure considered in the current paper. Thanks to the linearity of the payoff, the optimal stopping problem on the right-hand side of (2.16) is expressed in terms of a time homogeneous diffusion $\Phi$ with an affine payoff function independent of time. Note that this is the case not only for $\varepsilon=0$ but also for all possible parameter values.

In view of (2.16), we introduce the auxiliary optimal stopping problem

$$
\Gamma(t, z)=\sup _{0 \leq \tau \leq T-t} E^{*}\left[e^{\left(\mu_{l}-r\right) \tau}\left(1+Z_{\tau}\right)\right]
$$

where

$$
Z_{u}:=z \exp \left\{\left(\sigma \omega-\frac{\omega^{2}}{2}\right) u+\omega W_{u}\right\}, \quad u \geq 0
$$


and the supremum is taken over stopping times with respect to the filtration generated by $W$. Note that

$$
V=\frac{X_{0} \Gamma\left(0, \Phi_{0}\right)}{1+\Phi_{0}}
$$

Moreover, an optimal stopping time for the problem (2.20) translates to an optimal stopping time for the original problem (2.2).

In the next section we study the optimal stopping problem (2.20). In particular, we prove the existence of a continuous and monotonically increasing function $b:[0, T] \rightarrow[0, \infty)$ such that the stopping time

$$
\tau_{t, z}^{*}:=\inf \left\{u \in[0, T-t]: Z_{u} \leq b(t+u)\right\} \wedge(T-t)
$$

is an optimal stopping time, that is, a stopping time for which the supremum in (2.20) is attained. The following result is then a direct consequence of relation (2.18).

Theorem 2.5. Let $b$ be the function described above, the existence of which is proved in Proposition 3.2. Define the stopping time

$$
\tau^{*}=\inf \left\{t: X_{t} \leq \frac{X_{0}}{\Phi_{0}^{\beta}} e^{\varepsilon t} b^{\beta}(t)\right\} \wedge T .
$$

Then $\tau^{*}$ attains the supremum in (2.2).

Remark 2.6. The optimal stopping boundary and the optimal stopping time $\tau^{*}$ are illustrated in Figure 1. Note that it also follows from the analysis of the auxiliary problem below (in particular (3.25)) and relation (2.22) that $V$ is the solution of a free boundary problem. Indeed, straightforward calculations show that $V=U\left(0, \Phi_{0}\right)$, where the function $U(t, \phi)$ satisfies

$$
\begin{gathered}
U_{t}+\frac{\omega^{2} \phi^{2}}{2} U_{\phi \phi}+\frac{\phi(1+\phi)\left(\mu_{h}-\mu_{l}\right)+\omega^{2} \phi^{2}}{1+\phi} U_{\phi}+\frac{\phi\left(\mu_{h}-r\right)+\mu_{l}-r}{1+\phi} U=0 \quad \text { if } \phi>b(t), \\
U(t, \phi)=X_{0} \quad \text { if } \phi \leq b(t) \text { or } t=T \\
U_{\phi}(t, \phi)=0 \quad \text { if } \phi=b(t) .
\end{gathered}
$$

Remark 2.7. Note that the value $V$ exhibits an easy monotone dependence on the model parameters $\mu_{l}, \mu_{h}, r, \Pi_{0}$, and $T$. The dependence on volatility is slightly more involved to be analysed. However, it is a consequence of Corollary 2.7 in [11] that $\Gamma$ is monotonically increasing in the diffusion coefficient (i.e., in $\omega=\left(\mu_{h}-\mu_{l}\right) / \sigma$ ), so $V$ is decreasing in $\sigma$. The intuition behind this is that, in the case of a small volatility, learning of the true value of the drift is fast, which is beneficial for the agent. 


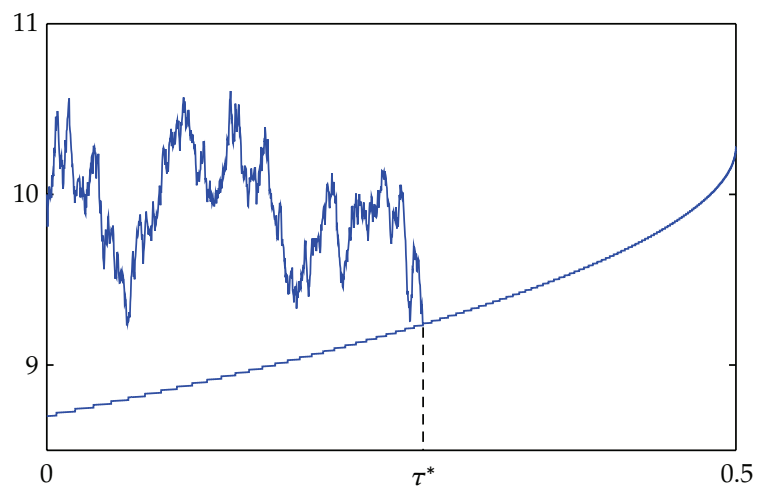

Figure 1: The optimal stopping boundary $\left(X_{0} / \Phi_{0}^{\beta}\right) e^{\varepsilon t} b^{\beta}(t)$, a simulated path of the asset price $X$, and the optimal stopping time $\tau^{*}$. We used the parameter values $\sigma=0.3, \mu_{h}=0.5, \mu_{l}=-0.3, r=0.1, T=0.5$, $X_{0}=10, \Pi_{0}=0.5$.

\section{The Auxiliary Optimal Stopping Problem}

In this section we study the optimal stopping problem (2.20). This problem is similar to the one arising in the valuation of American put options; compare [12] and Chapter 2.7 in [13]. We prove the existence of a monotone and continuous optimal stopping boundary, and we show that the boundary and the value function $\Gamma$ solves a related free boundary problem.

Recall that $Z$ satisfies

$$
\frac{d Z_{u}}{Z_{u}}=\sigma \omega d u+\omega d W_{u}=\left(\mu_{h}-\mu_{l}\right) d u+\omega d W_{u}, \quad u \geq 0
$$

We will also use the representation $Z_{u}=z H_{u}$, where

$$
H_{u}=\exp \left\{\left(\sigma \omega-\frac{\omega^{2}}{2}\right) u+\omega W_{u}\right\}
$$

With this notation,

$$
\Gamma(t, z)=\sup _{0 \leq \tau \leq T-t} E^{*}\left[e^{\left(\mu_{l}-r\right) \tau}\left(1+z H_{\tau}\right)\right]
$$

Proposition 3.1. The function $\Gamma:[0, T] \times(0, \infty) \rightarrow(0, \infty)$ is continuous.

Proof. Assume that $z_{2}>z_{1}>0$, and let $\tau$ be an optimal stopping time for $\Gamma\left(t, z_{2}\right)$ in the sense that

$$
\Gamma\left(t, z_{2}\right)=E^{*}\left[e^{\left(\mu_{l}-r\right) \tau}\left(1+z_{2} H_{\tau}\right)\right]
$$


International Journal of Stochastic Analysis

(such an optimal stopping time exists, see e.g., Theorem D.12 in [13]). Then we have

$$
\begin{aligned}
0 & \leq \Gamma\left(t, z_{2}\right)-\Gamma\left(t, z_{1}\right) \\
& \leq E^{*}\left[e^{\left(\mu_{l}-r\right) \tau}\left(1+z_{2} H_{\tau}\right)\right]-E^{*}\left[e^{\left(\mu_{l}-r\right) \tau}\left(1+z_{1} H_{\tau}\right)\right] \\
& =\left(z_{2}-z_{1}\right) E^{*}\left[e^{\left(\mu_{l}-r\right) \tau} H_{\tau}\right] .
\end{aligned}
$$

Ito's formula gives that the process $Y_{t}:=e^{\left(\mu_{l}-r\right) t} H_{t}$ satisfies

$$
d Y_{t}=\left(\mu_{h}-r\right) Y_{t} d t+\omega Y_{t} d W_{t}
$$

Since the drift $\left(\mu_{h}-r\right)$ is strictly positive, $e^{\left(\mu_{l}-r\right) t} H_{t}$ is a submartingale, so the Optional Sampling Theorem gives that

$$
0 \leq \Gamma\left(t, z_{2}\right)-\Gamma\left(t, z_{1}\right) \leq\left(z_{2}-z_{1}\right) E^{*}\left[e^{\left(\mu_{l}-r\right)(T-t)} H_{T-t}\right]=\left(z_{2}-z_{1}\right) e^{\left(\mu_{h}-r\right)(T-t)},
$$

which shows that $\Gamma$ is Lipschitz continuous in $z$.

Let $0 \leq t_{1}<t_{2}<T$ and $z \in(0, \infty)$. Let $\tau_{1}$ denote an optimal stopping time for $U\left(t_{1}, z\right)$, and set $\tau_{2}=\tau_{1} \wedge\left(T-t_{2}\right)$. Hence, $0 \leq \tau_{1}-\tau_{2} \leq t_{2}-t_{1}$. We then have

$$
\begin{aligned}
0 & \leq \Gamma\left(t_{1}, z\right)-\Gamma\left(t_{2}, z\right) \\
& \leq E^{*}\left[e^{\left(\mu_{l}-r\right) \tau_{1}}\left(1+z H_{\tau_{1}}\right)\right]-E^{*}\left[e^{\left(\mu_{l}-r\right) \tau_{2}}\left(1+z H_{\tau_{2}}\right)\right] \\
& =E^{*}\left[e^{\left(\mu_{l}-r\right) \tau_{1}}-e^{\left(\mu_{l}-r\right) \tau_{2}}\right]+z E^{*}\left[e^{\left(\mu_{l}-r\right) \tau_{1}} H_{\tau_{1}}-e^{\left(\mu_{l}-r\right) \tau_{2}} H_{\tau_{2}}\right] .
\end{aligned}
$$

Now

$$
\begin{aligned}
E^{*}\left[e^{\left(\mu_{l}-r\right) \tau_{1}} H_{\tau_{1}}-e^{\left(\mu_{l}-r\right) \tau_{2}} H_{\tau_{2}}\right] & =E^{*}\left[e^{\left(\mu_{l}-r\right) \tau_{2}} H_{\tau_{2}} E^{*}\left[e^{\left(\mu_{l}-r\right)\left(\tau_{1}-\tau_{2}\right)} \frac{H_{\tau_{1}}}{H_{\tau_{2}}}-1 \mid \mathcal{F}_{\tau_{2}}\right]\right] \\
& \leq E^{*}\left[e^{\left(\mu_{l}-r\right) \tau_{2}} H_{\tau_{2}}\right] F\left(t_{2}-t_{1}\right) \\
& \leq e^{\left(\mu_{h}-r\right)\left(T-t_{2}\right)} F\left(t_{2}-t_{1}\right)
\end{aligned}
$$

where

$$
F(t):=E^{*}\left[\sup _{u \in[0, t]} e^{\left(\mu_{h}-r-\omega^{2} / 2\right) u+\omega W_{u}}\right]-1
$$

and where we used the fact that $e^{\left(\mu_{l}-r\right) t} H_{t}$ is a submartingale. Note that $F(t) \rightarrow 0$ as $t \rightarrow 0$, which implies that the second term on the right-hand side of (3.8) tends to zero as $t_{2}-t_{1} \rightarrow 0$. 
A similar argument applies to the first term in (3.8), thus showing that $\Gamma$ is continuous as a function of $t$. Since $\Gamma$ is also uniformly continuous with respect to $z$, this finishes the proof.

Choosing the stopping time $\tau=0$ in (2.20), we find that $\Gamma(t, z) \geq G(z):=1+z$. Define the continuation region $\mathrm{C}$ by

$$
\mathcal{C}=\{(t, z) \in[0, T) \times(0, \infty): \Gamma(t, z)>G(z)\}
$$

and the stopping region $\Phi$ by

$$
\Phi=\{(t, z) \in[0, T] \times(0, \infty): \Gamma(t, z)=G(z)\} .
$$

According to general theory for optimal stopping problems, see, for example, [6], the stopping time

$$
\tau_{\Phi}=\inf \left\{0 \leq s \leq T-t:\left(t+s, Z_{s}\right) \in \Phi\right\}
$$

is an optimal stopping time in (2.20). Therefore, to determine an optimal stopping time, it suffices to determine the optimal stopping region $\Phi$.

Define $f(z):=\mathcal{L} G(z)-\left(r-\mu_{l}\right) G(z)$, where

$$
\mathcal{L} G(z):=\frac{\omega^{2} z^{2}}{2} G_{z z}(z)+\left(\mu_{h}-\mu_{l}\right) z G_{z}(z)
$$

is the infinitesimal operator of $Z$. A simple calculation shows that

$$
f(z)=\left(\mu_{h}-r\right) z+\mu_{l}-r \begin{cases}\geq 0 & \text { if } z \geq \frac{r-\mu_{l}}{\mu_{h}-r} \\ <0 & \text { if } z<\frac{r-\mu_{l}}{\mu_{h}-r}\end{cases}
$$

It therefore follows from Ito's formula that $e^{\left(\mu_{l}-r\right) s} G\left(Z_{s}\right)$ is a submartingale for $s \leq \inf \left\{u: Z_{u}<\right.$ $\left.\left(r-\mu_{l}\right) /\left(\mu_{h}-r\right)\right\}$. By the Optional Sampling Theorem, all points $(z, t)$ with $z>\left(r-\mu_{l}\right) /\left(\mu_{h}-r\right)$ belong to the continuation region $\mathcal{C}$.

A better bound for the stopping region $\Phi$ is easily derived by comparing $\Gamma$ with the corresponding "European value". More precisely, we have that

$$
\begin{aligned}
\Gamma(z, t) & =\sup _{0 \leq \tau \leq T-t} E^{*}\left[e^{\left(\mu_{l}-r\right) \tau}\left(1+Z_{\tau}\right)\right] \\
& \geq E^{*}\left[e^{\left(\mu_{l}-r\right)(T-t)}\left(1+Z_{T-t}\right)\right] \\
& =e^{\left(\mu_{l}-r\right)(T-t)}+z e^{\left(\mu_{h}-r\right)(T-t)}
\end{aligned}
$$

Therefore, all points $(z, t)$ such that $z>\left(1-e^{\left(\mu_{l}-r\right)(T-t)}\right) /\left(e^{\left(\mu_{h}-r\right)(T-t)}-1\right)$ satisfy $\Gamma(z, t)>$ $1+z$; that is, they belong to the continuation region. Note that the function $b^{E}(t):=(1-$ $\left.e^{\left(\mu_{l}-r\right)(T-t)}\right) /\left(e^{\left(\mu_{h}-r\right)(T-t)}-1\right)$ is increasing and satisfies $b^{E}(-\infty)=0$ and $b^{E}(T)=\left(r-\mu_{l}\right) /\left(\mu_{h}-r\right)$. 
Proposition 3.2. There exists a nondecreasing and right continuous function $b:[0, T) \rightarrow[0,(r-$ $\left.\left.\mu_{l}\right) /\left(\mu_{h}-r\right)\right]$ such that

$$
\mathcal{C}=\{(t, z) \in[0, T) \times(0, \infty): z>b(t)\} .
$$

Moreover, the supremum in (2.20) is attained for the stopping time

$$
\tau_{D}=\inf \left\{0 \leq u \leq T-t: Z_{u} \leq b(t+u)\right\} .
$$

Proof. First note that

$$
\mathcal{C}=\{(t, z): \Gamma(t, z)>G(z)\}=\{(t, z): \Gamma(t, z)>1+z\} .
$$

For some fixed $t \in[0, T)$ and $z^{\prime}>z>0$, suppose that $(t, z)$ is in $\mathcal{C}$. Then there exists a stopping time $\tau$ such that

$$
E^{*}\left[e^{\left(\mu_{l}-r\right) \tau}\left(1+z H_{\tau}\right)\right]>1+z
$$

Consequently,

$$
\begin{aligned}
\Gamma\left(t, z^{\prime}\right) & \geq E^{*}\left[e^{\left(\mu_{l}-r\right) \tau}\left(1+z^{\prime} H_{\tau}\right)\right] \\
& =E^{*}\left[e^{\left(\mu_{l}-r\right) \tau}\left(1+z H_{\tau}\right)\right]+\left(z^{\prime}-z\right) E^{*}\left[e^{\left(\mu_{l}-r\right) \tau} H_{\tau}\right] \\
& >1+z+\left(z^{\prime}-z\right) E^{*}\left[e^{\left(\mu_{l}-r\right) \tau} H_{\tau}\right] .
\end{aligned}
$$

Since the process $Y_{t}:=e^{\left(\mu_{l}-r\right) t} H_{t}$ is a submartingale, compare (3.6), the Optional Sampling Theorem gives

$$
\begin{aligned}
\Gamma\left(t, z^{\prime}\right) & >1+z+\left(z^{\prime}-z\right) E^{*}\left[e^{(\varepsilon-r) \tau} H_{\tau}\right] \\
& \geq 1+z+z^{\prime}-z=1+z^{\prime} .
\end{aligned}
$$

Therefore, $\left(t, z^{\prime}\right)$ also belongs to the continuation region $\mathcal{C}$, proving the existence of a function $b:[0, T) \rightarrow[0, \infty]$ such that

$$
\mathcal{C}=\{(t, z): t \in[0, T), z>b(t)\}
$$

The fact that $b$ only takes values smaller than $\left(r-\mu_{l}\right) /\left(\mu_{h}-r\right)$ follows from the discussion before Proposition 3.2, and the monotonicity of $b$ follows from the monotonicity of $t \mapsto \Gamma(t, z)$. Finally, the right continuity of $b$ follows from the fact that the continuation region $\mathcal{C}$ is an open set, and the optimality of $\tau_{D}$ is already established. 
Remark 3.3. In view of the discussion preceding Proposition 3.2, the optimal stopping boundary $b(t)$ satisfies

$$
b(t) \leq b^{E}(t)=\frac{\left(1-e^{\left(\mu_{l}-r\right)(T-t)}\right)}{\left(e^{\left(\mu_{h}-r\right)(T-t)}-1\right)} .
$$

A similar bound is then valid also for the optimal stopping boundary in Theorem 2.5.

Proposition 3.4. The value function $\Gamma(t, z)$ satisfies the boundary value problem

$$
\begin{gathered}
\Gamma_{t}(t, z)+\mathcal{L} \Gamma(t, z)+\left(\mu_{l}-r\right) \Gamma(t, z)=0 \quad \text { if } z>b(t), \\
\Gamma(t, z)=G(z)=1+z \quad \text { if } z \leq b(t) \text { or } t=T, \\
\Gamma_{z}(t, z)=G^{\prime}(z)=1 \quad \text { if } z=b(t) .
\end{gathered}
$$

Proof. Since $\Gamma(t, z)>1+z$ for $z>b(t)$ and $\Gamma(t, b(t))=1+b(t)$ for $t \in[0, T]$, it follows that

$$
\liminf _{\rho \searrow 0} \frac{\Gamma(t, b(t)+\rho)-\Gamma(t, b(t))}{\rho} \geq 1
$$

Thus, it remains to show that

$$
\limsup _{\rho \searrow 0} \frac{\Gamma(t, b(t)+\rho)-\Gamma(t, b(t))}{\rho} \leq 1 .
$$

For any $\rho>0$, denote by $\tau_{\rho}:=\tau_{t, b(t)+\rho}^{*}$ the optimal stopping time for the starting point $(t, b(t)+$ $\rho$ ) as defined in (2.23). We have

$$
\begin{aligned}
\Gamma(t, b(t)+\rho)-\Gamma(t, b(t)) & \leq E^{*}\left[e^{(\mu l-r) \tau_{\rho}}\left(1+(b(t)+\rho) H_{\tau_{\rho}}\right)\right]-E^{*}\left[e^{\left(\mu_{l}-r\right) \tau_{\rho}}\left(1+b(t) H_{\tau_{\rho}}\right)\right] \\
& =\rho E^{*}\left[e^{\left(\mu_{l}-r\right) \tau_{\rho}} H_{\tau_{\rho}}\right]
\end{aligned}
$$

We know that the optimal stopping boundary $s \mapsto b(s)$ is increasing on $[t, T]$ and $s \mapsto(\omega / 2-$ $\sigma) s$ is a lower function of the Brownian motion $W$ at zero. It follows that $\tau_{\rho} \rightarrow 0 P^{*}$-a.s. as $\rho \rightarrow 0$, which tells us that

$$
E^{*}\left[e^{\left(\mu_{l}-r\right) \tau_{\rho}} H_{\tau_{\rho}}\right] \longrightarrow 1
$$

as $\rho \rightarrow 0$ by the dominated convergence theorem. Hence, (3.27) holds, so $z \mapsto \Gamma(t, z)$ is $C^{1}$ at $z=b(t)$, and $\Gamma_{z}=1=G^{\prime}$.

The proof that $\Gamma$ satisfies the partial differential equation in (3.25) relies on the continuity of $\Gamma$ and follows along the same lines as, for example, in the case of the American put option, compare page 72 in [13]. We omit the details. 
Remark 3.5. Furthermore, it can be shown that the pair $(\Gamma, b)$ is the unique solution to the free boundary problem (3.25) (within some appropriate class of functions). We leave this and instead refer to Chapter 2.7 in [13], where this is shown for the American put option.

Proposition 3.6. The boundary $b(t)$ is continuous on $[0, T)$ and $b(T-)=\left(r-\mu_{l}\right) /\left(\mu_{h}-r\right)$.

Proof. It follows from Proposition 3.2 that $b$ is right continuous on $[0, T)$. To prove the leftcontinuity, define $b(T)=\left(r-\mu_{l}\right) /\left(\mu_{h}-r\right)$, and assume that the boundary $b(t)$ has a jump at $t_{*} \in(0, T]$, that is, $b\left(t_{*}\right)>b\left(t_{*}-\right)$.

By (3.15) and a continuity argument, there exists a $\delta<0$ and a one-side open rectangle $R:=\left[t^{\prime}, t_{*}\right) \times[c, d] \subseteq \mathcal{C}$ with $b\left(t_{*}-\right) \leq c<d<b\left(t_{*}\right)$ such that

$$
\begin{gathered}
\mathcal{L} G(z)-\left(r-\mu_{l}\right) G(z)<\delta<0, \\
0 \leq \Gamma_{z}(t, z)-G_{z}(t, z)<\frac{-\delta}{\left(\mu_{h}-\mu_{l}\right) b\left(t_{*}\right)}
\end{gathered}
$$

for all $(t, z) \in R$. Since $R$ is contained in $\mathcal{C}$, we also have

$$
L \Gamma(t, z)-\left(r-\mu_{l}\right) \Gamma(t, z)=-\Gamma_{t}(t, z) \geq 0
$$

Together with (3.30), this yields

$$
\left(\mu_{h}-\mu_{l}\right) z\left(\Gamma_{z}(t, z)-G_{z}(t, z)\right)+\frac{1}{2} \omega^{2} z^{2}\left(\Gamma_{z z}-G_{z z}\right)=L \Gamma-L G \geq\left(r-\mu_{l}\right)(\Gamma-G)-\delta
$$

Using (15), it follows that

$$
\Gamma_{z z}-G_{z z} \geq 2 \omega^{-2} z^{-2}\left(r-\mu_{l}\right)(\Gamma-G) \geq 2 \omega^{-2} b\left(t_{*}\right)^{-2}\left(r-\mu_{l}\right)(\Gamma-G)=: \eta>0
$$

in $R$. Therefore,

$$
\begin{aligned}
\Gamma(t, z)-G(z) & =\int_{b(t)}^{z} \int_{b(t)}^{u}\left(\Gamma_{z z}(t, v)-G_{z z}(v)\right) d v d u \\
& \geq \frac{1}{2} \eta(z-b(t))^{2} \geq \frac{1}{2} \eta\left(c-b\left(t_{*}-\right)\right)^{2}>0
\end{aligned}
$$

for any $(t, z)$ in the rectangle. Since both the value and the gain functions are continuous, this leads to $\Gamma\left(t_{*}, z\right)>G(z)$ for any $z \in[c, d]$, which contradicts the fact that $\left(t_{*}, z\right)$ is in the stopping region. Therefore, $b(t)$ is continuous on $t \in[0, T)$ and $b(T-)=\left(r-\mu_{l}\right) /\left(\mu_{h}-r\right)$.

\section{An Integral Equation for the Optimal Stopping Boundary}

In this section we derive an integral equation for the optimal stopping boundary. The derivation follows along similar lines as for the American put option; see [12]. 
Theorem 4.1. The optimal stopping boundary $b(t)$ satisfies the integral equation

$$
\begin{aligned}
1+b(t)= & e^{\left(\mu_{l}-r\right)(T-t)}+b(t) e^{\left(\mu_{h}-r\right)(T-t)} \\
& -\int_{0}^{T-t}\left(\mu_{l}-r\right) e^{\left(\mu_{l}-r\right) u} N\left(\frac{1}{\omega \sqrt{u}}\left[\ln \frac{b(t+u)}{b(t)}-\omega \sigma u+\frac{\omega^{2} u}{2}\right]\right) \\
& +b(t)\left(\mu_{h}-r\right) e^{\left(\mu_{h}-r\right) u} N\left(\frac{1}{\omega \sqrt{u}}\left[\ln \frac{b(t+u)}{b(t)}-\omega \sigma u-\frac{\omega^{2} u}{2}\right]\right) d u,
\end{aligned}
$$

where $N(x)=(1 / \sqrt{2 \pi}) \int_{-\infty}^{x} e^{-y^{2} / 2} d y$ is the cumulative distribution function of the standard normal distribution.

Proof. Fix a $t \in[0, T]$ and $Z_{0}=z \in(0, \infty)$. Applying Ito's formula to $e^{\left(\mu_{l}-r\right) s} \Gamma\left(t+s, Z_{s}\right)$ and taking the expected value give

$$
\begin{aligned}
e^{\left(\mu_{l}-r\right)(T-t)} E^{*}\left[G\left(Z_{T-t}\right)\right] & =e^{\left(\mu_{l}-r\right)(T-t)} E^{*}\left[\Gamma\left(T, Z_{T-t}\right)\right] \\
& =\Gamma(t, z)+\int_{0}^{T-t} e^{\left(\mu_{l}-r\right) u} E^{*}\left[F\left(Z_{u}\right) I\left(Z_{u} \leq b(t+u)\right)\right] d u,
\end{aligned}
$$

where $G(y)=1+y$ and $F=\mathcal{L} G-\left(r-\mu_{l}\right) G$ as before. (The use of Ito's formula can be motivated by similar arguments as for the American put option; cf. [14].) Straightforward calculations give

$$
e^{\left(\mu_{l}-r\right)(T-t)} E^{*}\left[G\left(Z_{T-t}\right)\right]=E^{*}\left[e^{\left(\mu_{l}-r\right)(T-t)}\right]+E^{*}\left[e^{\left(\mu_{l}-r\right)(T-t)} Z_{T-t}\right]=e^{\left(\mu_{l}-r\right)(T-t)}+z e^{\left(\mu_{h}-r\right)(T-t)} .
$$

The integrand of the right-hand side in (4.2) is

$$
\begin{aligned}
e^{\left(\mu_{l}-r\right) u} E^{*}\left[F\left(Z_{u}\right) I\left(Z_{u} \leq b(t+u)\right)\right]= & e^{\left(\mu_{l}-r\right) u} z\left(\mu_{h}-r\right) E^{*}\left[H_{u} I\left(Z_{u} \leq b(t+u)\right)\right] \\
& +e^{\left(\mu_{l}-r\right) u}\left(\mu_{l}-r\right) E^{*}\left[I\left(Z_{u} \leq b(t+u)\right)\right] .
\end{aligned}
$$

We have

$$
\begin{aligned}
& E_{t, z}^{*}\left[H_{u} I\left(Z_{u} \leq b(t+u)\right)\right] \\
& =E_{t, z}^{*}\left[\exp \left[\left(\sigma \omega-\frac{\omega^{2}}{2}\right) u+\omega W_{u}\right] I\left(\frac{W_{u}}{\sqrt{u}} \leq \frac{\ln (b(t+u) / z)+\left(\omega^{2} / 2-\sigma \omega\right) u}{\omega \sqrt{u}}\right)\right] \\
& =e^{\sigma \omega u} N\left(d_{1}\right),
\end{aligned}
$$

where

$$
d_{1}=\frac{1}{\omega \sqrt{u}}\left[\ln \frac{b(t+u)}{z}-\omega \sigma u-\frac{\omega^{2} u}{2}\right]
$$


Similarly

$$
E_{t, z}^{*}\left[I\left(Z_{u} \leq b(t+u)\right)\right]=N\left(d_{2}\right),
$$

where $d_{2}=d_{1}+\omega \sqrt{u}$. Using (4.2)-(4.7) and inserting $z=b(t)$ yield the integral equation (4.1).

Remark 4.2. Using local time-space calculus, it was proved in [14] that the optimal stopping boundary of the American put option is the unique solution to the corresponding integral equation. Using similar techniques, uniqueness for (4.1) can be established. We omit the details.

\section{Closing a Short Position}

In this section we consider an agent with a short position in the asset and who seeks an optimal time to close the position. To study this situation we formulate the optimal stopping problem

$$
v=\inf _{0 \leq \tau \leq T} E\left[e^{-r \tau} X_{\tau}\right]
$$

where the infimum is taken over $\mathcal{F}^{X}$-stopping times $\tau$. All the assumptions about the model are as described in Section 2.

By exactly the same arguments provided above, we find that

$$
v=\frac{X_{0}}{1+\Phi_{0}} \gamma\left(0, \Phi_{0}\right)
$$

where $\gamma$ is defined through the auxiliary optimal stopping problem

$$
\gamma(t, z)=\inf _{0 \leq \tau \leq T-t} E^{*}\left[e^{\left(\mu_{l}-r\right) \tau}\left(1+Z_{\tau}\right)\right]
$$

where

$$
Z_{u}:=z \exp \left\{\left(\sigma \omega-\frac{\omega^{2}}{2}\right) u+\omega W_{u}\right\}, \quad u \geq 0
$$

and the infimum is taken over stopping times with respect to the filtration generated by $W$. Moreover, an optimal stopping time for the problem (5.3) translates to an optimal stopping time for the original problem (5.1).

The following results parallel those for the optimal liquidation problem for a long position, and the proofs are omitted.

Theorem 5.1. There exists a nonincreasing and continuous function $b:[0, T) \rightarrow\left[\left(r-\mu_{l}\right) /\left(\mu_{h}-\right.\right.$ $r), \infty)$ with $b(T-)=\left(r-\mu_{l}\right) /\left(\mu_{h}-r\right)$ such that the continuation region $\mathcal{C}=\{(x, t) \in[0, T) \times(0, \infty)$ : $\gamma(t, z)<1+z\}$ satisfies

$$
\mathcal{C}=\{(t, z) \in[0, T) \times(0, \infty): z<b(t)\} .
$$


Moreover, the infimum in (5.3) is attained for the stopping time

$$
\tau_{D}:=\inf \left\{0 \leq u \leq T-t: Z_{u} \geq b(t+u)\right\}
$$

The function $\gamma:[0, T] \times(0, \infty) \rightarrow(0, \infty)$ is continuous and satisfies the boundary value problem

$$
\begin{gathered}
\gamma_{t}(t, z)+\mathcal{L} \gamma(t, z)+\left(\mu_{l}-r\right) \gamma(t, z)=0 \quad \text { if } z<b(t) \\
\gamma(t, z)=G(z)=1+z \quad \text { if } z \geq b(t) \text { or } t=T \\
\gamma_{z}(t, z)=G^{\prime}(z)=1 \quad \text { if } z=b(t) .
\end{gathered}
$$

The optimal stopping boundary $b(t)$ satisfies the integral equation

$$
\begin{aligned}
1+b(t)= & e^{\left(\mu_{l}-r\right)(T-t)}+b(t) e^{\left(\mu_{h}-r\right)(T-t)} \\
& -\int_{0}^{T-t}\left(\mu_{l}-r\right) e^{\left(\mu_{l}-r\right) u}\left\{1-N\left(\frac{1}{\omega \sqrt{u}}\left[\ln \frac{b(t+u)}{b(t)}-\omega \sigma u+\frac{\omega^{2} u}{2}\right]\right)\right\} \\
& +b(t)\left(\mu_{h}-r\right) e^{\left(\mu_{h}-r\right) u}\left\{1-N\left(\frac{1}{\omega \sqrt{u}}\left[\ln \frac{b(t+u)}{b(t)}-\omega \sigma u-\frac{\omega^{2} u}{2}\right]\right)\right\} d u .
\end{aligned}
$$

Corollary 5.2. The infimum in (5.1) is attained for the stopping time

$$
\tau^{*}:=\inf \left\{t: X_{t} \geq \frac{X_{0}}{\Phi_{0}^{\beta}} e^{\varepsilon t} b^{\beta}(t)\right\} \wedge T .
$$

Remark 5.3. Unlike the optimal liquidation problem for a long position, the problem of this section also makes sense to study with an infinite horizon.

\section{Acknowledgment}

E. Ekström gratefully acknowledges the support from the Swedish Research Council (VR).

\section{References}

[1] J. Evans, V. Henderson, and D. Hobson, “Optimal timing for an indivisible asset sale," Mathematical Finance, vol. 18, no. 4, pp. 545-567, 2008.

[2] V. Henderson and D. Hobson, "An explicit solution for an optimal stopping/optimal control problem which models an asset sale," Annals of Applied Probability, vol. 18, no. 5, pp. 1681-1705, 2008.

[3] J. du Toit and G. Peskir, "Selling a stock at the ultimate maximum," Annals of Applied Probability, vol. 19, no. 3, pp. 983-1014, 2009.

[4] S. E. Graversen, G. Peskir, and A. N. Shiryaev, "Stopping Brownian motion without anticipation as close as possible to its ultimate maximum," Theory of Probability and its Applications, vol. 45, no. 1, pp. 41-50, 2000.

[5] R. S. Liptser and A. N. Shiryayev, Statistics of Random Processes I General Theory, Springer, New York, NY, USA, 1977. 
[6] G. Peskir and A. Shiryaev, Optimal Stopping and Free-Boundary Problems, Lectures in Mathematics ETH Zürich, Birkhäuser, Basle, Switzerland, 2006.

[7] I. Karatzas and X. Zhao, "Bayesian adaptive portfolio optimization," in Option Pricing, Interest Rates and Risk Management, Handbook of Financial Mathematics, pp. 632-669, Cambridge University Press, Cambridge, UK, 2001.

[8] P. Lakner, "Utility maximization with partial information," Stochastic Processes and Their Applications, vol. 56, no. 2, pp. 247-273, 1995.

[9] J.-P. Décamps, T. Mariotti, and S. Villeneuve, "Investment timing under incomplete information," Mathematics of Operations Research, vol. 30, no. 2, pp. 472-500, 2005.

[10] M. Klein, "Comment on "investment timing under incomplete information"," Mathematics of Operations Research, vol. 34, no. 1, pp. 249-254, 2009.

[11] E. Ekström, "Properties of American option prices," Stochastic Processes and their Applications, vol. 114, no. 2, pp. 265-278, 2004.

[12] S. Jacka, "Optimal stopping and the American put," Mathematical Finance, vol. 1, no. 2, pp. 1-14, 1991.

[13] I. Karatzas and S. E. Shreve, Methods of Mathematical Finance, vol. 39 of Applications of Mathematics, Springer, New York, NY, USA, 1998

[14] G. Peskir, "On the American option problem," Mathematical Finance, vol. 15, no. 1, pp. 169-181, 2005. 


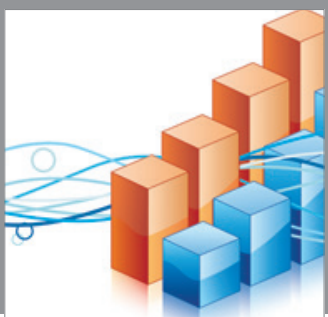

Advances in

Operations Research

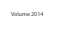

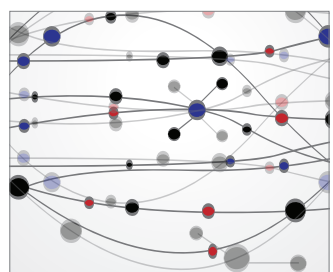

\section{The Scientific} World Journal
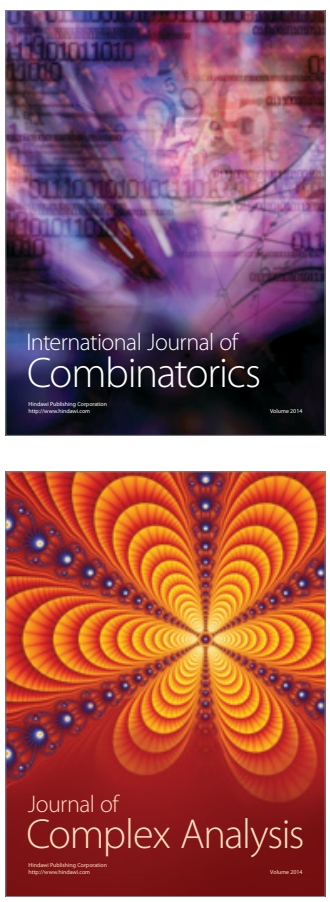

International Journal of

Mathematics and

Mathematical

Sciences
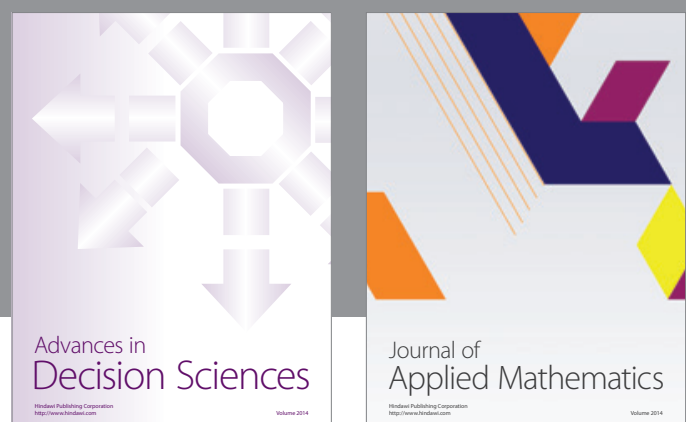

Journal of

Applied Mathematics
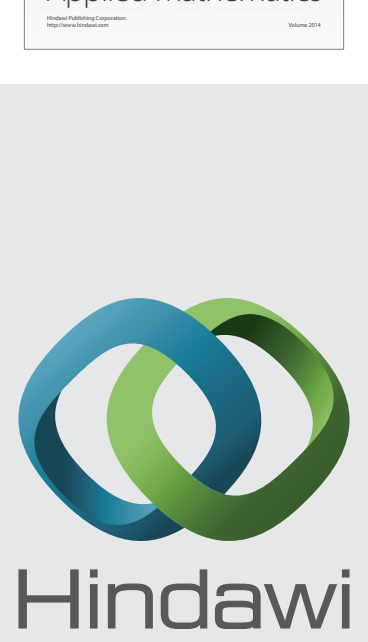

Submit your manuscripts at http://www.hindawi.com
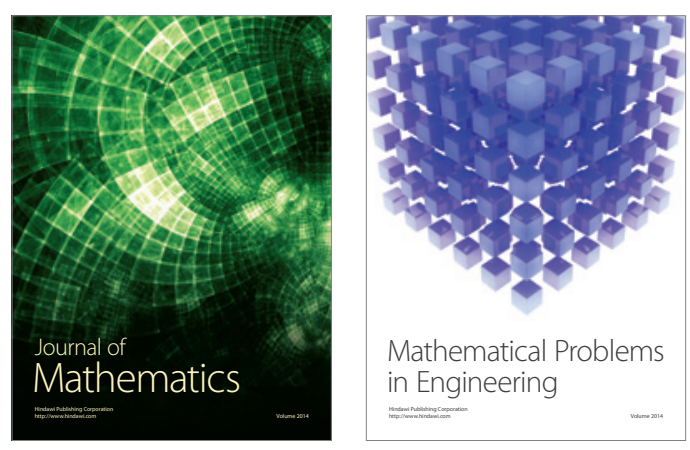

Mathematical Problems in Engineering
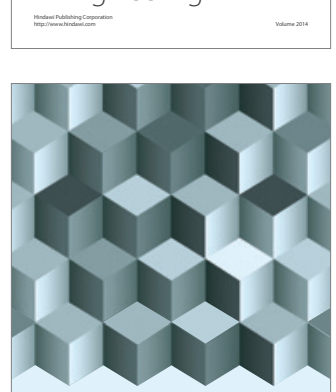

Journal of

Function Spaces
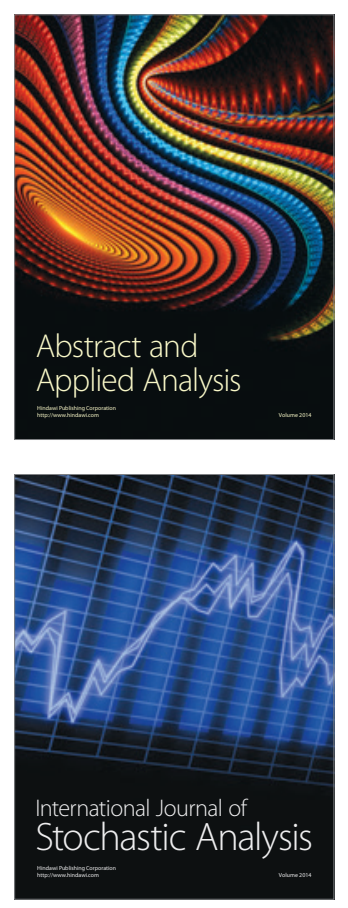

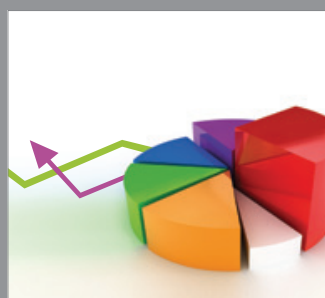

ournal of

Probability and Statistics

Promensencen
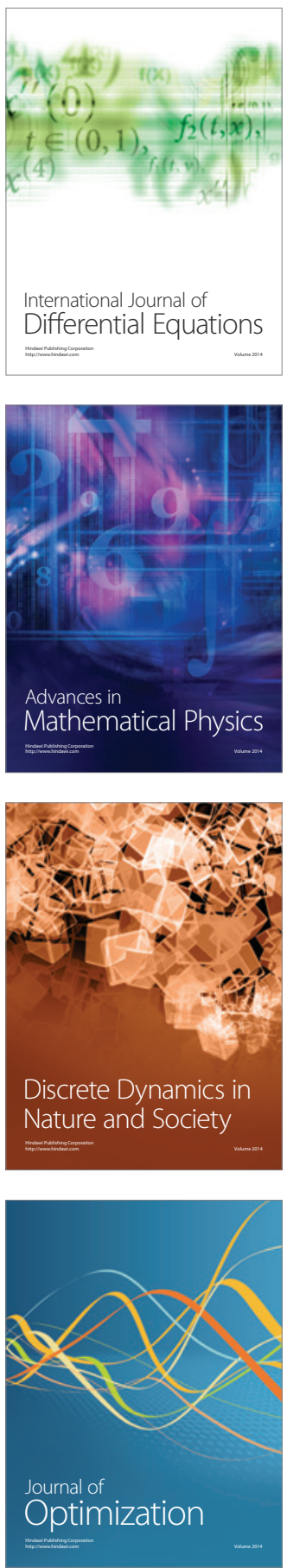